Editorial

\title{
Dental Management in Coronavirus Disease 2019 Outbreak
}

\author{
Arvind Babu Rajendra Santosh ${ }^{1}$ \\ ${ }^{1}$ Faculty of Medical Sciences, School of Dentistry, University of the \\ West Indies, Mona, Kingston, Jamaica
}

Eur Dent Res Biomater J 2020;1:1-3

Dentists are doctors of oral health, whose main aims are to relieve pain from the tooth and associated structures and to restore dental function and esthetics. The primary objective of dental practice is prevention and promotion strategies related to oral and overall health. However, during the coronavirus disease 2019 (COVID-19) outbreak, dentists should focus on the prevention of COVID-19 transmission as well as other important objectives of dental management. Based on the current situation, the shift of dental management is driven to dental emergency cases, with the focus to prevent transmission of COVID-19. It would be a better choice to either avoid or reschedule nonemergency dental treatment.

COVID-19 is a rapidly growing health concern due to its alarming spread across nations and a significant potential to cause severe respiratory problems in few cases. ${ }^{1}$ Evidently, the transmission of infection is through the respiratory droplets from symptomatic COVID-19 patients and asymptomatic COVID-19 infected individuals. The incubation period of COVID-19 virus is estimated as 5 to 6 days; however, the commonly adopted duration is 14 days for health observation and quarantine of exposed persons. ${ }^{2}$ The transmission of COVID-19 includes direct transmission such as cough sneeze, droplet inhalation, and contact with the oral, nasal, and ocular mucosal membranes. ${ }^{3}$ The symptoms of the COVID-19 include fever, dry cough, shortness of breath, and other medical presentations. The diagnosis of COVID-19 can be based on a combination of clinical symptoms, CT (computed tomography) imaging findings, laboratory test such as swab test, and reverse transcriptase-polymerase chain reaction for molecular diagnosis. The reports suggest that all age groups are susceptible to COVID-19; however, those who are in close contact with a COVID-19 positive patient are at a high risk of contracting the COVID-19 infection. Based on the observation from China, the fatality rate is 0.39 to $4.05 \%$, which is lower than that of SARS (severe acute

respiratory syndrome) and MERS (Middle East respiratory syndrome). ${ }^{2}$ The risk of cross-contamination is high in dental practice due to exposure to droplets and aerosols from dental procedures. ${ }^{4-6}$ Recent reports have mentioned that oral symptoms of COVID-19 could be dryness of mouth and loss of taste sensation. ${ }^{7}$ Consistent detection of COVID-19 pathogen is detected in the salivary gland ductal epithelium earlier than respiratory tissues. ${ }^{8}$ Hence salivary detection for COVID-19 need to be tested for detection of asymptomatic or COVID-19 infection. ${ }^{9}$ Hence, managing a dental patient during COVID-19 should be done with strict prevention and infection control guidelines. Hence, managing a dental patient during COVID-19 should be done with strict prevention and infection control guidelines.

Dental students of undergraduate category and dental observers should be advised to either not be an integral part or have limited involvement in the patient management in the dental settings at this situation. This is suggested due to the novel nature of infectious disease, and experts are trying to understand the management strategies with available resources. However, the management criteria policies of infectious diseases shall be guided to students through learning portals using audio-video technology such as live-telecasting, and the staff should take a lead in managing dental patients.

The following suggestions are based on currently available evidence, but few are narrative opinion:

- Routine or nonemergency dental practices should be either suspended or minimized to prevent the spread of infection via droplets or aerosols. ${ }^{2}$

- Dental emergencies should be treated strictly under the recommended infection control program in dental office setting.

- All dental procedures performed in the office, as well as the data regarding patients, patient attenders, and number of family members living with the patients, phone

\section{Address for correspondence}

Arvind Babu Rajendra Santosh, BDS, MDS,

Faculty of Medical Sciences, School of Dentistry,

University of the West Indies, Mona, Kingston, Jamaica

(e-mail: arvindbabu.rajendrasantosh@uwimona.edu.jm).
DOI https://doi.org/ C2020 Dental Investigation Society License terms 10.1055/s-0040-1710411.

() (1) $\ominus \circledast$ 
numbers, and addresses should be carefully documented. This data will be helpful to track the COVID-19 infection status either through the dentist to patient or the patient to dentist when required.

- During patient scheduling through phone communication, the receptionist must take information such as most recent travel and acute respiratory tract infection related symptoms. Encourage the patients who positively respond to travel or medical history questions to seek medical attention immediately. Similarly, all dental patients who received dental treatment should be guided about the symptoms related to COVID-19. Follow-up phone communication should be made postoperatively on 3,6 , or 14 days in case of any patient with COVID-19 symptoms. Patients who report acute respiratory tract related symptoms should be immediately medically attended.

- Strict prescreening protocols, case selection, and pretreatment risk assessment should be performed for screening COVID-19 infected individuals. ${ }^{2}$

- Strictly avoid nonemergency dental and elective dental procedure for high-risk individuals, such as, patients in the geriatric age group, individuals with comorbidities and/or respiratory conditions, immunocompetent individuals, immunocompromised individuals, or individuals who received a recent transplant procedure unless there is need for dental care is functionally impeding general health.

- The receptionist should welcome the patients and guide them about hand sanitizing and recommended self-hygiene care. The office should facilitate hand sanitizing and tissue papers to all individuals who visit the dental office. ${ }^{4}$

- Symptomatic patients visiting the dental office should be provided with N95 or FFP2 standard masks. Symptomatic patients should be referred to a designated medial practitioner in the dental setting, and a guidance letter for managing dental patients from the medical practitioner should be requested for. ${ }^{4}$

- The dentist should thoroughly communicate with the patients regarding the need for emergency versus nonemergency dental treatment during the COVID-19 outbreak. Dental patient education along with pamphlets can help in spreading information about the right dental health management among family and friends of dental patients.

- Prior to dental management, consultation should be made with the designated medical practitioner to embrace a multidisciplinary approach for dental problems and produce socially accountable practice. ${ }^{2}$

- Appropriate referral should be made to a fellow dental office or a specialist dental office when the condition cannot be managed in the current settings.

- The most common dental emergencies are severe tooth pain, tooth avulsion, uncontrolled bleeding following tooth extraction, swelling, space infection, and jaw bone fracture.

- Dentist and dental assistants who are involved in the treatment that generate aerosols must wear personal protective equipment (PPE) such as masks (N95 or FFP2 standard masks), hand-hygiene, gloves, gowns, goggles, and face shields. ${ }^{4}$
- Dental surgeons and assistants should be cautioned about mouth breathing while with or without mouth/face masks. Breathing through the mouth may attract aerosol-related infection and should be avoided.

- Dental treatment should be performed in a well-ventilated room. Following the treatment, the room should be disinfected, kept vacant, and well ventilated. ${ }^{2}$

- Minimize the number of patient intake in the dental settings. Appropriate break time should be facilitated while managing multiple patients on the same day.

- The patient waiting area should not be crowded but well ventilated, hand sanitizers and tissue papers should be provided, and the seating area should be well spaced with a minimum of 3-feet distance.

- Four-handed dentistry is effective for infection control. ${ }^{2,6}$

- Pre-examination or preoperational procedure should begin with mouth rinse that contains oxidative diluted with $1 \%$ hydrogen peroxide or $0.2 \%$ povidone should be performed. ${ }^{5}$

- During the examination of the oral cavity, check for any soreness in the throat and cough spots (reddened areas) in the posterior palatal mucosa.

- All extractions should be evaluated for the possibility of suturing to minimize postoperative complications, and resorbable sutures must be preferred to minimize postoperative visits. $^{2}$

- Procedures that involve a production of aerosols should be either avoided or minimized to dental emergencies. High or low saliva ejectors can reduce droplets or aerosol infection to some extent. ${ }^{2,6}$

- Patients should not be left with saliva ejectors or suction tips either turned off or on in the oral cavity when procedures are not performed. This is suggested to prevent cross-contamination from the saliva ejectors or suction tips.

- Procedures that have a tendency to induce cough, such as dental impression procedures, and intraoral radiographs should be minimized unless it is an emergency. Orthopantomography should be preferred in dental emergency patients. $^{2}$

- Following dental impression procedures, the impression should be washed thoroughly and dipped in $60 \%$ alcohol for 3 to 5 minutes.

- During oral prophylaxis or routine periodontal treatment, the procedure should be performed using hand instruments and mechanical therapy where essentially required.

- Rubber dams should be used in operative restorative procedures in all cases. General caution related to rubber dams should be taken to prevent allergies.

- Although airotors are used in routine caries management, hand instruments should be used for caries removal where possible.

- Dental emergency cases with a known history of COVID-19 should be treated in an isolated clinic room within the office. Patients should be educated regarding quarantining and dental management. However, extreme care should be taken to avoid discrimination related conflicts such as treating COVID-19 versus non-COVID-19 patients. 
- Dental prescriptions should be given to patients following medical doctors' consultation to prevent any drug-drug interactions.

- All patients and any accompanying person who visits the dental office should be evaluated for temperature prior to routine patient observation at the dental chair. ${ }^{2}$

- Dental staff members should be regularly evaluated, and medical consultation and swab test should be performed when necessary based on the evidence of incubation period of COVID-19 symptoms. Flu vaccine can be recommended among dental staff members and referred to a designated medical practitioner for getting the vaccination.

- When any of the dental staff members is diagnosed positively for COVID-19, he/she should be withdrawn from all duties of the dental office. Strict self-quarantine and medical management should be recommended. All patients attended by such dental staff member should be retrospectively monitored and examined for medically.

- Rostering dental staff member on a weekly basis will also allow the dental office management to track clinical symptoms if any.

- Biowaste management should be strictly followed, ${ }^{2}$ and data entry related to the same should be reviewed by the chief dentist of the office. Dental patients should be guided about the disposal of gauze or mouth mask when provided. Fumigation of dental office should be done as guided by the recommended infection control practices.

- All relevant information related to COVID-19 infection experience gained in your dental settings should be documented and submitted to the Ministry of Health and Wellness in a periodic manner for social accountability during the COVID-19 outbreak.

Due to the highly contagious nature of COVID-19, PPE must be used in dental treatment to prevent aerosol mediated transmission. During this pandemic outbreak, the dentist must focus on emergency treatment and suspend all nonemergency and routine dental care. This approach will minimize the risk of transmission of COVID-19 virus from dental offices. In addition, it will also address the concerns of acute crisis of PPE among medical health care professionals.

\section{Conflict of Interest}

None declared.

\section{References}

1 World Health OrganizationRolling updates on coronavirus disease (COVID-19). Available at: https://www.who.int/emergencies/diseases/novel-coronavirus-2019/events-as-theyhappen. Accessed March, 2020

2 Meng L, Hua F, Bian Z. Coronavirus disease 2019 (COVID-19): emerging and future challenges for dental and oral medicine. J Dent Res 2020. Doi:10.1177/0022034520914246

3 Lu C-W, Liu XF, Jia ZF. 2019-nCoV transmission through the ocular surface must not be ignored. Lancet 2020;395(10224): e39

4 World Health Organization. The COVID-19 risk communication package for healthcare facilities. Available at: http://iris. wpro.who.int/handle/10665.1/14482. Accessed March, 2020

5 Peng X, Xu X, Li Y, Cheng L, Zhou X, Ren B. Transmission routes of 2019-nCoV and controls in dental practice. Int J Oral Sci 2020;12(1):9

6 Li RW, Leung KW, Sun FC, Samaranayake LP. Severe acute respiratory syndrome (SARS) and the GDP. Part II: implications for GDPs. Br Dent J 2004;197(3):130-134

7 Ren YF, Rasubala L, Malmstrom H, Eliav E. Dental Care and Oral Health under the Clouds of COVID-19. JDR clinical and translational research. 2020:2380084420924385

8 To KK, Tsang OT, Chik-Yan Yip C, et al. Consistent detection of 2019 novel coronavirus in saliva [published online ahead of print, 2020 Feb 12]. Clin Infect Dis 2020:ciaa149. Doi: 10.1093/ cid/ciaa149

9 Arvind Babu RS, Krishnamurthy K, Ramana Reddy BV. Proposal of Research Model for the Detection of COVID-19 among Asymptomatic Carriers. International Archives of Otorhinolaryngology 2020. Ahead of press. DOI: https://doi.org/ 10.1055/ s-0040-1712936 